\title{
La educación artística y su rol en la atención educativa a la diversidad
}

\section{The Artistic Education and its Roll in the Educative Attention to the Diversity}

\author{
Antonio Bouza López \\ Universidad Central "Marta Abreu" de Las Villas, Santa Clara, Cuba \\ ORCID: https://orcid.org/0000-0002-8381-9817 \\ Beatriz Rodríguez Rodríguez \\ Universidad Central "Marta Abreu" de Las Villas, Santa Clara, Cuba \\ ORCID: https://orcid.org/0000-0002-1318-5425 \\ Yoani Delgado Pérez \\ Universidad Central "Marta Abreu" de Las Villas, Santa Clara, Cuba \\ ORCID: https://orcid.org/0000-0003-0206-713X
}

Received 02-12-20 Revised 04-25-20 Accepted 09-13-20 On line 09-29-20

*Correspondence

Email: eseraggon@gmail.com
Cite as:

Bouza, A., Rodríguez, B., \& Delgado, Y. (2020). La educación artística y su rol en la atención educativa a la diversidad. Propósitos y Representaciones, 8 (SPE3), e737. Doi: http://dx.doi.org/10.20511/pyr2020.v8nSPE3.737

(C) Universidad San Ignacio de Loyola, Vicerrectorado de Investigación, 2020. 


\section{Resumen}

La investigación "La Educación Artística y su rol en la atención educativa a la diversidad", responde a la necesidad de darle solución a la carencia de herramientas cognoscitivas y prácticas para el trabajo con la diversidad desde la formación inicial del educador artístico, partiendo de la propia concepción para la formación de este profesional, quien deberá utilizar la apreciación y creación artística como herramientas para solucionar problemáticas educativas más allá de la clase, el taller y la actividad comunitaria. En la investigación se parte de un diagnóstico que permitió detectar el estado del problema y posibilitó constatar que, en el componente académico de esta carrera, se carece de herramientas cognoscitivas artístico-prácticas para el tratamiento educativo de la diversidad. El trabajo investigativo permite el logro de transformaciones en la formación inicial de los estudiantes, como son: aprehensión de conocimientos para el trabajo con adolescentes y jóvenes en esta arista educativa, demostrándose así, las potencialidades del arte como una alternativa para la atención educativa a la diversidad.

Palabras clave: Educación artística; Formación; Atención educativa a la diversidad.

\section{Summary}

The investigation "the Artistic Education and its roll in the educative attention to the diversity", responds to raise theoretical foundations that guarantee the necessity of cognoscitivas and practical tools for the work with the diversity from the initial formation of the artistic educator, starting off of the own conception of the formation of this professional future, using the appreciation and artistic creation like tool to solve problematic educative beyond the class, the factory and the communitarian activity. For the same part of a diagnosis that allowed to detect the state of the problem and made possible to state that in the academic component of this student, artistic-practices for the educative treatment of the diversity are lacked cognoscitivas tools. With the implementation of this investigation transformations in the initial formation of the students will be obtained, as they are: apprehension of knowledge for the work with adolescents and young people in this educative edge, demonstrating itself thus, the potentialities of the art like an alternative for the educative attention to the diversity.

Keywords: Artistic Education; Formation; Educative Attention to the Diversity.

\section{Introducción}

El mundo actual atraviesa por distintas problemáticas relacionadas con la falta de entendimiento hacia lo diverso, conflictos raciales, culturales, religiosos, biológicos e informacionales marcan estas tendencias desde los ámbitos globales hasta los particulares. Es inconcebible pensar que en pleno siglo XXI, luego de trazarse acuerdos, tratados y leyes internacionales en pos del respeto mutuo de cada cuál tal como existe, piensa y vive continúen tales problemas.

En la Conferencia Mundial sobre Necesidades Educativas Especiales, celebrada en Salamanca, en 1994, se dio un gran impulso a la educación integradora. Las recomendaciones de la Conferencia se fundaron en el principio de la integración, basado en que las escuelas deben acoger a todos los niños, independientemente de sus condiciones físicas, intelectuales, sociales, emocionales, lingüísticas u otras. Deben acoger a niños discapacitados y niños bien dotados, a niños que viven en la calle y que trabajan, niños de poblaciones remotas o nómadas, niños de 
minorías lingüísticas, étnicas o culturales y niños de otros grupos o zonas desfavorecidos o marginados.

El principio de atención a la diversidad está basado en la obligación de los Estados y sus Sistemas Educativos a garantizar a todos el derecho a la educación (Dieterlen, 2001; Gordon, 2001). En Cuba, la educación es fiel exponente ante el mundo del desarrollo cultural de un pueblo, la cual tiene como misión fundamental la formación integral de la personalidad. El gobierno revolucionario y sus instituciones socializadoras han llevado a cabo innumerables acciones para el trabajo educativo con la diversidad, ejemplo de lo cual, son la creación de las escuelas especiales, las metodologías, resoluciones, programas televisivos, spot, propaganda en los medios de comunicación masiva para la labor desde y hacia lo diverso, a pesar de lo cual, no se puede decir con exactitud que se está ajeno a la problemática de entender y trabajar eficazmente con la diversidad en todo su sentido.

La atención educativa a la diversidad se ha programado a través de directrices metodológicas para su implementación con calidad desde las instituciones pertinentes, aunque no siempre se ejecuta de la manera adecuada, porque evidentemente el ser humano, que a su vez es ente ejecutor en esta delicada y dedicada tarea, no es perfecto.

El ejemplo más cercano a ello es el educador artístico, quien, en su formación inicial no recibe directamente todos los conocimientos necesarios acerca de las habilidades cognoscitivas práctico-artísticas para la atención educativa a la diversidad, se comprueba, además, que el mayor protagonismo a recaído casi siempre en las manifestaciones de artes plásticas y música con estos fines, y no en el teatro y la danza siendo soportes indirectos de dicha actividad.

Precisamente para determinar fundamentos que contribuyan a entender cómo se da el proceso de formación inicial en la educación superior, sus particularidades y potencialidades respecto a la atención educativa a la diversidad, se realizó la revisión bibliográfica relacionada con el tema.

La formación inicial de los docentes es un tema cada vez más recurrente, por comportarse como un proceso complejo e interesante, teniendo el mismo un rol protagónico en cuanto a políticas del estado, manifestadas en desarrollo sociocultural y educativo. Amplios son los referentes teóricos en relación, y en los cuales se presentan puntos de convergencia. Repetidamente autores como; Minsun Kim, Andrews y Carr, (2004), Imbernón (1998) Marcelo y Vaillant (2009), en el ámbito internacional iberoamericano, que plantean la formación del docente como un proceso de formación permanente, de constante aprendizaje científico, técnico y sociocultural, psicológico, disciplinar y didáctico, en el cual estos saberes van conformando las formas en que debe aproximarse a su desempeño en la práctica.

Estos autores sostienen que la formación del docente comienza durante el período de su formación inicial en los centros formadores y continúa durante toda la vida profesional. Este período se concibe como paso a la preparación para enfrentar todos los contextos socioculturales en que trabajarán, la diversidad de sus alumnos, las situaciones conflictivas en el aula y las relaciones con los colegas de la escuela. 
La formación inicial del docente, entendido como agente del desarrollo profesional debe, dotar de un bagaje sólido en el ámbito cultural, psicopedagógico y personal, ha de capacitar al futuro profesor o profesora para asumir la tarea educativa en toda su complejidad, actuando con la flexibilidad y la rigurosidad necesaria, esto es, apoyando sus acciones en una fundamentación válida. (Imbernón, 1998).

En Cuba la conceptualización de la formación inicial ha sido abordada de igual manera por ilustres pedagogos:

Álvarez, C. (2009) la define como un proceso totalizador que agrupa los procesos educativos, desarrolladores e instructivos. Según López (2002) se interpreta como base del desarrollo y consecuencia de este, orientada hacia el logro de los objetivos de la educación. De manera general se concibe como el conjunto de actividades organizadas con el objetivo de proporcionar los conocimientos, destrezas y disposiciones necesarias para que el docente pueda desempeñarse en la práctica de manera consciente, sobre todo, con relación a la implicación en decisiones que han de tomarse en ella.

Por otro lado, Horruitiner (2009) declara que el proceso de formación profesional tiene como objetivo preparar integralmente al estudiante en una determinada carrera universitaria y abarca tanto los estudios de pregrado como los de posgrado, objetivo que puede ser alcanzado con la adecuada integración de los procesos (dimensiones o funciones) ya abordados. A saber, y para especificar su esencia, es necesario abundar en que la dimensión instructiva, que posibilita el contacto del estudiante con el objeto de su profesión, expresa cómo este adquiere los conocimientos como contenidos de la cultura y habilidades esenciales de su profesión y se prepara para emplearlas al desempeñarse como tal, en un determinado puesto de trabajo.

En cuanto a la dimensión educativa refleja la esencia de la labor educativa en el proceso de formación integral y es asumida por los docentes desde el contenido de las asignaturas. Promueve la formación de valores que regulan el comportamiento de los estudiantes, para que sean hombres útiles socialmente, comprometidos con su realidad y aptos para actuar sobre ella, transformarla y hacerla más humana. Por último, la dimensión desarrolladora enfatiza en que los estudiantes forman competencias para asegurar un desempeño profesional exitoso, a través de la vinculación del estudio y el trabajo, en la que se emplearán los conocimientos, habilidades, capacidades y valores asumidos para dar solución a los problemas profesionales que se les presenten en su actividad laboral.

Al analizar cada una de las posturas teóricas en cuanto a la formación inicial se constata que la misma es un proceso no solo de andamiajes teóricos para la futura labor, sino que es una vía práctica para sembrar valores éticos- estéticos, modos de actuación, reflexiones sobre el componente educativo en el que se encontrará y propuestas de transformación del medio desde lo diverso y hacia el mismo.

La formación del educador artístico se dirige a la preparación de un profesional competente para la articulación apreciativa del arte, como un gestor cultural dentro del centro educacional y fuera del mismo, como un crítico cualificado, le proporciona un bagaje cultural amplio, le propicia un desarrollo de conocimientos y habilidades con los cuales deberá trabajar y reflexionar desde la práctica de los mismos. 
Hace 5 años se retomó por parte del Ministerio de Educación Superior la carrera de Licenciatura en Educación. Educación Artística, con el grandísimo reto de formar profesionales que desde la apreciación artística lograrán consolidar esa meta de convertir a la escuela en el centro cultural más importante de la comunidad, y así, continuar la ardua tarea de desprender de nuestra sociedad fenómenos ajenos como la banalidad, la chapuza y los antivalores éticos y estéticos.

El estudiante que hoy se forma en la carrera tendrá que enfrentarse luego de concluir sus estudios de pregrado a un perfeccionamiento de la educación cubana, donde la atención educativa a la diversidad es protagónica.

A partir de la revisión de los documentos normativos de la carrera, estrategias curriculares, programas de asignatura se pudo constatar que, dentro del componente académico artístico, el estudiante no recibe herramientas desde su formación, que les permitan hacer del arte una vía eficaz para comprender y ejecutar la atención educativa a la diversidad.

Insuficientes son las investigaciones que abordan dicho tema, donde el arte y el educador artístico como puntal de procesos transformadores en la escuela logran hechos significativos en la atención educativa a la diversidad, aunque es válido resaltar que desde la empírea varias son las experiencias positivas que se dan en diferentes escuelas de nuestro país.

El educador artístico es un ente educativo y social de vital importancia siempre que su dimensión es aprovechada lógica y organizadamente, pues su componente de acción laboral no solo reside en la escuela, sino que va hacia la comunidad, familia y sociedad.

Este es capaz desde la apreciación artística incentivar el buen gusto, las buenas normas de conducta y crear puentes que generan procesos culturales, artísticos y estéticos que garantizan desde la práctica hechos sociales capaces de llegar a todos los contextos cotidianos. A partir de estos importantes hallazgos nace la necesidad de aportar acciones educativas que desde la formación laboral investigativa apoyadas en las expresiones artísticas evidencien en la práctica la importancia de la preparación en cuanto a la atención educativa a la diversidad de los estudiantes de la carrera Licenciatura en Educación. Educación Artística.

\section{La atención educativa a la diversidad.}

El Diccionario Filosófico plantea que la diversidad es la variedad y diferencia que se presenta en la realidad de los objetos y sujetos que actúan en ella. El hombre es diverso en tanto ser, biológicopsíquico-social. El reto de la sociedad actual es que la convivencia adquiera unos altos y positivos niveles entre sus habitantes. Por un lado, la población proviene de países diferentes, a la par que personas con distintas capacidades y talentos, junto con las diferencias habituales entre los seres humanos componen una sociedad de "diferentes" que obliga a aprender a vivir en el respeto a esa diferencia, buscando en ella motivos y causas de enriquecimiento para todos. Por tanto, la atención a la diversidad consiste en aplicar un modelo de educación que consiste en ser capaz de ofrecer a cada alumno la ayuda pedagógica que él necesite, ajustando la intervención educativa a la individualidad del alumnado: esta aspiración no es otra que adaptar la enseñanza a las diferentes 
capacidades, intereses y motivaciones del alumnado. (Araque Hontangas y Barrio de la Puente, 2010), se considera además que:

Por ser diferentes aprendemos de manera diferente, con diferente ritmo y calidad, incluso algunos podemos tener limitaciones o desventajas por múltiples causas (orgánicoconstitucionales, fisiológicas, socioculturales), por lo que aprendemos más lentamente, con ciertas dificultades y necesitamos más ayudas, pero eso no disminuye en nada nuestra condición de seres humanos, ni niega la posibilidad de aprender y acceder al desarrollo." (López, 2002, p.12).

La Doctora Argelia Fernández Díaz en su artículo "Algunas consideraciones sobre diversidad educativa" del año 2005, expresa diferentes reflexiones del tema:

- Las diferencias individuales están condicionadas por la forma de manifestación de las premisas biológicas, psicológicas y sociales, así como la historia de vida de cada sujeto.

- Las fuentes de la diversidad están en la interrelación entre el plano externo y el interno, en relación con el contexto en que actúa el sujeto, (a través de la actividad y la comunicación.).

- Además, plantea diferentes acciones para potenciar su desarrollo desde lo educativo:

- Acciones que fomentan la igualdad de oportunidades entre hombres y mujeres (la desigualdad por razón de género vista como una cuestión general que afecta a la totalidad de la población);

- Acciones que van a la eliminación de las desigualdades que resultan de otros factores (discapacidad física o mental; discriminación racial, cultural, étnica o religiosa; situación de desventaja socioeconómica, ritmos de aprendizaje, capacidad intelectual, entre otras)

Esto indica que según la autora la atención a la diversidad debe cubrir, entre otras las siguientes necesidades:

- Cubrir las necesidades de las personas con algún defecto discapacitante, (de cualquier naturaleza). Esto está relacionado con la accesibilidad física y pedagógica del contenido de la enseñanza, medidas de acompañamiento o de apoyo ya sea por los profesores u otro personal, uso de nuevas tecnologías adaptándolas a los diferentes tipos de discapacidad, garantizar su movilidad con condiciones adecuadas (evitar las barreras arquitectónicas, entre otros). Todo esto, si es posible, dentro de un grupo de estudiantes con un desarrollo adecuado, para ganar en la elevación de la autoestima personal de los discapacitados.

- Contribuir a la igualdad entre los diferentes géneros: Esto se aplica no sólo a la igualdad en el acceso a actividades orientadas en todos los sectores de la educación, sino también a fomentar la igualdad de oportunidades entre hombres y mujeres por medios educativos. Lo que se puede expresar en la eliminación de los estereotipos sexuales en el material didáctico, la diversificación de los ámbitos de estudio que eligen las mujeres y los que eligen los hombres y fomentar una mejor orientación vocacional que conduzca al aumento de la empleabilidad (Ciencias y Tecnologías), Además de la educación para el acceso a cargos por parte de las mujeres, entre otros.

- Contribuir a la lucha contra el racismo. Se fomenta de diversos modos la educación intercultural y las medidas destinadas a promover el respecto y el entendimiento de la diversidad étnica, cultural y lingüística, de acuerdo con la especificidad de las diferentes 
acciones del programa. También se impulsarán medidas para estimular la participación plena y activa de personas de todos los grupos étnicos y lingüísticos.

- Contribuir a contrarrestar los efectos de las situaciones de desventaja socioeconómica, principalmente fomentando la participación de personas y centros que la sufran mediante el proceso de selección y, en algunas acciones, mediante un mayor apoyo económico.

- El sistema educativo cubano se prepara con mayor intensidad para la atención educativa a la diversidad, fenómeno que, aunque no es totalmente nuevo para las instituciones educativas y se ha evidenciado desde los principios de la Educación en Cuba con el Triunfo de la Revolución, se aplica hace pocos años, si cierto es que muchas son las insatisfacciones que aún quedan en tal sentido, al mismo tiempo mucho se ha avanzado en el tema en el país.

- El sistema educativo, y en especial la escuela, debe crear condiciones para que exista igualdad de oportunidades para todos sus alumnos. El acceso a las instituciones educativas no significa necesariamente una respuesta adecuada a la igualdad de oportunidades y a la exclusión social. Todos los niños pueden tener éxito, aprender, desarrollarse, acceder al conocimiento y a la cultura, emplear sus habilidades intelectuales y prácticas y alcanzar el máximo desarrollo de sus capacidades. (López Machín, 2002).

La igualdad de oportunidades no implica "tratar de igual forma a todos", como han expresado algunos docentes y padres, sin mucha meditación, en las indagaciones que, hechas, o "trabajar por homogeneizar el grupo", "eliminar las diferencias", como han referido otros, lo cual, a nuestro juicio, no es lo que debemos proponernos, ni es posible lograrlo (López Machín, 2002).

Muchos más ejemplos pudieran ser abordados, a partir de la dimensión tan amplia del tema, aunque estos a partir de la concepción del autor pudieran ser fundamentales, especialmente el determinado para la Educación Artística, ya que, como elemento ético-estético, de libertad personal, de satisfacción grupal, se presenta como un instrumento educativo capaz de crear verdaderas transformaciones.

Varias son las fórmulas para que lo planteado desde el sistema educativo obtenga resultados positivos en el proceso pedagógico de manera general, el autor expone a continuación ejemplo de algunas:

- Escuelas y Comunidades Inclusivas en pensamientos, en acción y cambio.

- Personal Docente y no Docente preparado.

- Actividades organizadas en pos de Cultura hacia y para la Diversidad.

- La Educación Artística como herramienta eficaz.

\section{La Educación Artística y su rol en la atención educativa a la diversidad.}

La Educación Artística es un concepto del Siglo XIX y se reduce inicialmente a las Artes Plásticas y la Música, aunque en su propio desarrollo ha manifestado su práctica en las demás expresiones del arte. Además, esta conforma un par dialéctico con la Educación Estética. El Diccionario la define como: 
Formación de necesidades en relación con el arte, capacidad de apreciación y expresión o creación, de acuerdo a las aptitudes. No se reduce a lo curricular, debe apoyarse en la vida: vinculación de la escuela, con la familia y la comunidad.

El estado cubano y las instituciones educativas de nuestro país siempre han dedicado especial atención a la pericia de la Educación Artística conforme al proceso educativo de la escuela. La Educación Artística es capaz de desarrollar habilidades apreciativas y creativas en niños, jóvenes y adultos que les permiten no solo disfrutar del arte, sino, entender el medio que los rodea, definirlo desde el arte e impulsar cambios favorables al mismo. Esta fomenta lo identitario, aleja de lo banal, lo grosero, lo no adecuado, lo mal hecho; su dialogo con el individuo es hacia un entorno siempre positivo, donde el arte y la cultura son vías para el desarrollo.

El profesor e investigador cubano Pablo René Estévez en su libro "Los colores del Arcoíris" del año 2008, plantea:

"Genéricamente hablando, el hombre no ha logrado todavía "poner riendas a la fiera" que dormita dentro de sí y no ha podido, por lo tanto, construir relaciones estéticas universales para regir su comportamiento de acuerdo con las "leyes de la belleza" (Estévez, 2008, p. 25).

Es un reto, desgraciadamente, al alcance de pocos pueblos (el capitalismo, por su esencia inhumana, es el principal responsable por ello). Sin embargo, el pueblo cubano tiene una gran oportunidad para alcanzarlo por medio de la educación.

La Educación Artística no solo comprende en su finalidad el hecho de preparar al individuo para la apreciación y disfrute del arte, está encargada a promover valores, buenas conductas, respeto a sí mismo y a los demás, a convivir y ser parte el medio.

En las nuevas fórmulas de escuelas inclusivas, de atención educativa a la diversidad, la Educación artística juega un rol fundamental. En el contexto escolar, la formación artística del estudiantado "crea capacidades humanas inventivas y críticas para que el arte pueda seguir su trayectoria histórica de revelar, mediante los estilos y las formas estéticas de la pintura, escultura, música, danza, literatura y dibujo, los diversos entendimientos y sentimientos del ser humano sobre la naturaleza y sobre su propia existencia en el mundo" (Siqueira 2009).

Acha (2007) plantea que la educación artística al plantearse dentro de un ámbito educativo y sociocultural, introduce al estudiantado hacia una reconceptualización de los análisis, interpretación y valoración de los efectos que el dibujo, la pintura y la escultura, entre otros, estimularían la retroalimentación de las experiencias lúdicas. Asimismo, Fernández (2003) esboza que por medio de la creatividad "el niño desarrolla sus destrezas mentales de tal manera que el arte le sirve de trampolín para otras disciplinas”.

Prados (2004) plantea que los alumnos y alumnas asociados a sentimientos de inferioridad y baja autoestima bloquean su potencial creativo. Sin embargo, es posible de revertir esta situación, si en la práctica creadora, se realicen con periodicidad actividades artísticas encaminadas a superar las limitaciones significativas de la actividad adaptativa, que incluyan en su desarrollo las características escolares, motivacionales, de personalidad, oportunidades sociales y laborales, que en la acción educativa se transformen en "oportunidades y estímulos de 
manera que puedan expresarse confiadamente, superando inhibiciones y estimulando la espontaneidad".

Promover la salud mental y emocional por medio de la educación artística y el desarrollo de la creatividad en las personas diagnosticadas con Discapacidad Intelectual, ayuda a mejorar los factores ambientales del trabajo escolar relacionados con la insatisfacción, frustración y complejos de inferioridad; mejora las habilidades cognitivas y psicomotrices que estimulan la necesidad del hacer, el sentir y el pensar; fortalece la autoestima por medio de la retroalimentación de las habilidades interpersonales; fomenta la alegría de experimentar nuevos aprendizajes y acciones lúdicas derivadas de la imaginación creativa (Tilley, 1991), que conducen en su conjunto, a interactuar y sentirse parte activa de los centros socio-educativos donde están inmersos, "dándose presencia, separándose y uniéndose, perdiéndose y encontrándose, asumiendo lo existente pero interaccionado activamente en él, inscribiéndose en el espacio de la complejidad que es el vivir" (López \& Martínez, 2006).

En este trabajo se defiende la idea que si el estudiante de la licenciatura en Educación Artística desde su formación inicial recibe las herramientas teóricas y prácticas necesarias será capaz de atender con eficacia la diversidad de alumnos en su desempeño profesional.

\section{Metodología}

Se emplea la investigación-acción, Elliott (1990) plantea la necesidad de que los profesores mejoren su capacidad para generar conocimientos profesionales en vez de aplicar los desarrollados por otros especialistas. Aunque, como él mismo indica, las generalizaciones basadas en muestras pueden facilitar las reflexiones a los profesores, pero nunca sustituirlas.

En el sentido más amplio, podemos decir que implica una reflexión relacionada con el diagnóstico en un primer paso, donde se analizan los problemas y se plantean las hipótesis y una intervención donde se prueba un experimento de cambio aplicado a una realidad social (Cohen,1990).

tipo de investigación posibilitó desarrollar acciones artísticas con los estudiantes de la carrera Licenciatura en Educación. Educación Artística, a través de las expresiones artísticas las cuales se proyectan en la práctica desde la asignatura Formación Laboral Investigativa. Entre los métodos utilizados en el proceso investigativo se señalan el analítico - sintético, este se manifiesta en todo el procesamiento de la información obtenida a partir del proceso investigativo, la interpretación de los resultados y la fundamentación del sistema de acciones.

El inductivo-deductivo se utiliza en función del establecimiento de los referentes teóricos y metodológicos. El método histórico-lógico se evidencia en la búsqueda de los antecedentes El método de ascenso de lo abstracto a lo concreto se aplica para el proceso de elaboración teórico práctica de las diferentes definiciones que se asumen en la investigación.

Dentro de los métodos del nivel empírico se emplea el análisis de documentos, las entrevistas grupales, la observación. La triangulación metodológica permite comprobar la veracidad de los resultados. 


\section{Participantes}

La unidad de análisis estuvo constituida por todos los estudiantes de la carrera Licenciatura en Educación. Educación Artística. La Muestra de la investigación se obtuvo por un procedimiento probabilístico e intencional eligiéndose a 16 estudiantes que cursan el 3er Año de dicha carrera. Los criterios de selección se obtuvieron a partir de que las expresiones artísticas son protagónicas en este año, y que además el componente laboral se hace aún más directo y práctico en este año.

Tabla 1.

Caracterización de la Muestra

\begin{tabular}{ccl}
\hline Sexo & Promedio de Edad & Opción en la que solicitan la carrera \\
\hline $\begin{array}{c}\text { Femenino } \\
13\end{array}$ & $\begin{array}{l}8 \text { en } 1 \text { opción } \\
4 \text { en } 2 \text { opción } \\
1 \text { en } 3 \text { opción }\end{array}$ \\
$\begin{array}{c}\text { Masculino } \\
3\end{array}$ & 20 & 3 en 1 opción \\
\hline
\end{tabular}

\section{Resultados}

En el análisis de documentos se pudo establecer que los documentos normativos de la carrera, la asignatura Expresiones Artísticas y la disciplina Formación Laboral Investigativa cuentan con potencialidades para la atención educativa a la diversidad. A partir de estas potencialidades se diseñan y orientan las acciones artísticas de atención educativa a la diversidad, a ejecutar y evaluar en el componente laboral. Componente Laboral que se realiza en la Secundaria Básica José Martí, teniendo como protagonistas a los educandos del 8 vo grado.

Se realiza la observación a una de las acciones artísticas de atención educativa a la diversidad comprobando que los educandos que presentan necesidades educativas especiales les cuesta trabajo participar en la actividad, los estudiantes de la carrera positivamente los incluyen a través de herramientas lúdicas, de animación y motivación proyectadas en las acciones, 12 de los estudiantes las realizan efectivamente para un $75 \%$ y 4 no utilizan de manera amplia y positiva las herramientas y acciones, para un $25 \%$.

La entrevista realizada a los estudiantes del 3er año de la carrera Licenciatura en Educación. Educación Artística, constato que según los entrevistados las acciones complementan aún más su diapasón cognoscitivo, estos entienden la diversidad no solo desde lo conceptual planteado en las acciones sino que efectivamente crean sus propias concepciones de comprensión y atención a la diversidad, antes de la propuesta y proyección de las acciones solo 3 conocían la conceptualización de diversidad, representando un 18,75\%, los 16 estudiantes se muestran sensibilizados con el tema para 100\%, 6 de ellos creen que las acciones pueden mejorar en cuanto a contenido, contextualización y práctica, representando un 37,5\% y los 6 restantes ven las acciones muy completas en cuanto a contenido y práctica, para un 62,5\%. La encuesta aplicada arrojo como resultados que: A los 16 estudiantes encuestados les gustan las expresiones artísticas como alternativa de atención educativa a la diversidad como parte del componente laboral, para 
un 100\%, solo 5 comprenden la importancia de la atención educativa a la diversidad en su futura labor y en el contexto educacional cubano, representando un $31,25 \%$.

\section{Discusión}

La investigación tuvo como objetivo brindarle soporte teórico-práctico desde las expresiones artísticas y teniendo como centro de acción la disciplina Formación Laboral Investigativa, en su componente práctico-laboral, de manera general a los estudiantes de la carrera Licenciatura en Educación. Educación Artística, y muy particular a los 16 estudiantes del 3er año de dicha carrera. Partiendo de un diagnóstico exploratorio que permitió identificar las fortalezas, debilidades y necesidades que poseían los documentos normativos de la carrera para la atención educativa a la diversidad, llevaron a confeccionar, organizar y ejecutar un sistema de acciones artísticas de atención educativa a la diversidad, que consolidarán la preparación del estudiante de la carrera en formación inicial para su inserción en diferentes contextos educativos.

Para De La Torre y Barrios (2002), formar es "ayudar a tomar conciencia de las propias actuaciones y como mejorarlas", o sea, la formación debe ser permanente, permitiendo al individuo realizar una reflexión de su práctica de manera que sea posible provocar cambios y actualizaciones constantes en su forma de estar y de actuar.

La elección de un modelo de formación de profesionales docentes debe entonces asentarse en una perspectiva holística de los conocimientos, de los objetivos y de las metas que en educación se orientan hacia el futuro, teniendo en cuenta los constantes cambios sociales y la consecuente necesidad de actualización de los conocimientos del individuo, con la mirada puesta en su desarrollo integral y en su inserción en el mundo laboral (Moya, 2018).

Este sistema de acciones proyectado desde el componente práctico-laboral del estudiante de la carrera Licenciatura en Educación. Educación Artística, en formación inicial, se plantea como una alternativa eficaz para su desarrollo integral cognoscitivo y práctico, no es esquemático, en su ejecución se puede flexibilizar en la medida que el entorno escolar lo exija, cuenta con 15 acciones que abarcan las diferentes manifestaciones del arte, en las cuales podemos encontrar acciones lúdicas, ejercicios de improvisación, expresión corporal, representación y libertad muscular, trabajos con la voz y dicción, técnicas manuales colectivas, etc. Las acciones se planificaron pensando en articularlas estilo taller, donde la interacción sea premisa, donde participen todos y los resultados se completen por todos en una actividad de cierre de la aplicación de este sistema de acciones.

Los resultados indican que el sistema de acciones artísticas de atención educativa a la diversidad aplicados en los estudiantes del 3er año de la carrera Licenciatura en Educación. Educación Artística, cuenta con adecuadas propiedades del arte y la atención educativa a la diversidad ejecutadas en el componente práctico-laborar que permiten considerarla como una propuesta válida, novedosa y confiable que puede aplicarse a los demás años de la carrera y en futuras investigaciones relacionadas con el tema. 


\section{Conclusiones}

Los resultados obtenidos, de modo general, revelan la necesidad de futuras investigaciones relacionadas con la educación artística y su rol en la atención educativa a la diversidad, sobre todo en la educación superior por su importancia en la formación general integral del individuo, y que estas permitan desarrollar propuestas bien concebidas conscientemente que integren universidad, componente laboral y comunidad, que apuesten por un sistema de actividades sin perder de vista los valores estéticos y éticos más auténticos, vistas desde el enriquecimiento del currículo de formación inicial de las carreras artísticas, particularizándolas en disciplinas como las expresiones artísticas, la extensión universitaria y el componente práctico-laboral.

Además, los resultados arrojaron potencialidades para el desarrollo del tema en cuestión, sobre todo teniendo en cuenta el significado de educador artístico, su función en la sociedad, la motivación que desprende la práctica del arte y la Educación Artística.

Asimismo, de cuán importante es la figura de este ente educativo en la escuela que hoy se encuentra en perfeccionamiento, no solo para articular procesos creativos desde el arte sino para potenciar experiencias positivas del arte en la atención educativa a la diversidad.

\section{Referencias}

Acha, J. (2007). Expresión y apreciación artísticas: Artes Plásticas, Reimpresión. México: Trillas.

Álvarez de Zayas, C. M. (1989). Didáctica: La escuela en la vida. La Habana: Editorial Pueblo y Educación.

Araque, N. \& Barrio, J.L. (2010). Atención a la diversidad y desarrollo de procesos educativos inclusivos. Revista Prisma Social, 4, 1-37.

Cohen, L. \& Manion, L. (1990). Métodos de investigación educativa. Madrid: La Muralla.

Dieterlen, P. (2001): Derechos, necesidades básicas y obligación institucional. En A. Ziccardi (comp.). Pobreza, desigualdad social y ciudadanía. Los límites de las políticas sociales en América Latina. Buenos Aires: CLACSO.

Elliott, J. (1990). La investigación-acción en educación. Madrid: Morata.

Estévez, P. (2008). Los colores del arco iris. La Habana: Editorial Pueblo y Educación.

Fernández, A. (2005). Algunas consideraciones sobre la diversidad educativa. La Habana: Editorial pueblo y Educación.

Fernández, M. (2003). Creatividad, arte terapia y autismo. Un acercamiento a la actividad Plástica como proceso creativo en niños autistas. Arte, individuo y sociedad, 15: 135152.

Gordon, S. (2001). Ciudadanía y derechos sociales: ¿criterios distributivos?. En A. Ziccardi (comp.). Pobreza, desigualdad social y ciudadanía. Los límites de las políticas sociales en América Latina. Buenos Aires: CLACSO.

Horruitiner, P. (2009). La universidad cubana: modelo de formación. La Habana: Ministerio de Educación Superior.

Imbernón, F. (1998). La Formación y el Desarrollo Profesional del Profesorado. España, Graõ, Serie Pedagogía. $3^{\text {a }}$ Edición.

López, R. (2002). Educación y diversidad. Texto; Convocados por la Diversidad. La Habana: Editorial Pueblo y Educación. 
López, R. \& Nieves, M. L. (2000). Educación de alumnos con necesidades educativas especiales: Fundamentos y actualidad. La Habana: Editorial Pueblo y Educación.

López, M. \& Martínez, N. (2006). Arteterapia. Conocimiento interior a través de la Expresión artística. Madrid.

Moya, Y. (2018). La preparación del instructor de arte en formación inicial para la dirección coral en la escuela primaria. Tesis de Especialización: Docencia en Psicopedagogía. Santa Clara. Villa Clara.

Prados, M. (2004). El taller de arteterapia en niños y niñas con retraso mental escolarizados en un centro específico. En N. Martínez \& M. Fernández (Coords.). Arteterapia y Educación (pp. 185-204). Madrid: Comunidad de Madrid,Consejería de Educación.

Siqueira, I. (2009). Nuevos campos de desarrollo y aplicación de la educación artística. En: L. Jiménez, I. Aguirre \& L. Pimentel (Coords.). Educación Artística, Cultura y Ciudadanía (pp. 145-154). Madrid: OIE. Recuperado: de www.oei.es/histórico/metas2021/EDART2.pdf

Tilley, P. (1991). El arte en la educación especial. Barcelona: CEAC.

UNESCO. (1994). Declaración y Marco de Acción sobre Educación para todos. Conferencia Mundial. Dakar, Senegal, 